\title{
Temporal Dermoid Cyst with a Partial Dermal Sinus Tract
}

\author{
Joseph A. Shehadi, Ibrahim A. Alorainy, Karen M. Johnston
}

\begin{abstract}
Background: Cranial dermal sinuses are rare and occur most frequently in the posterior fossa and along the midline. Likewise, supratentorial dermoid cysts are very uncommon. Method: We report a unique case of an adult female patient with both a supratentorial dermoid cyst and an incomplete dermal sinus tract. Results: The patient is a 31-year-old female, who presented with a new onset complex partial seizure. Neuroimaging and surgery revealed a right superior temporal dermoid cyst with an associated dermal sinus tract. Furthermore, the dermal sinus tract was incomplete and had no cutaneous manifestations. Conclusion: We present a rare patient with an off midline supratentorial dermoid cyst associated with a uniquely incomplete cranial dermal sinus tract. The dermal sinus tract involved the bone, dura and intradural compartment, without involving the overlying skin. This represents a novel variant in the spectrum of cranial dermal sinus abnormalities.
\end{abstract}

RÉSUMÉ: Kyste dermoïde temporal avec sinus dermique partiel: à propos d'un cas. Introduction: Les sinus dermiques crâniens sont rares et se retrouvent le plus souvent dans la fosse postérieure et sur le plan sagittal. Les kystes dermoïdes supratentoriels sont également très rares. Méthode: Nous rapportons le cas d'une femme adulte ayant un kyste dermoïde supratentoriel et un sinus dermique incomplet. Résultats: Il s'agit d'une femme âgée de 31 ans qui a consulté pour un phénomène récent de crise partielle complexe. La neuroimagerie et la chirurgie ont révélé l'existence d'un kyste dermoïde temporal supérieur droit associé à un sinus dermique. De plus, le sinus dermique était incomplet et ne comportait pas de manifestation cutanée. Conclusion: Nous présentons le cas rare d'une patiente présentant un kyste dermoïde supratentoriel non médian associé à un sinus dermique crânien incomplet. Le sinus dermique impliquait l'os, la dure-mère et le compartiment intradural, sans impliquer la peau sus-jacente. Ceci constitue une nouvelle variante dans le spectre des anomalies des sinus dermiques crâniens.

Can. J. Neurol. Sci. 1999; 26:321-324

Cranial dermal sinuses are quite rare, and fewer than 100 cases have been reported in the English literature since its original description by Ogle in 1865. ${ }^{1,2}$ Cranial dermal sinuses most frequently occur in the posterior fossa and along the midline..$^{2-4}$ We present a patient with both a cranial dermal sinus and associated dermoid cyst, which are non-midline and supratentorial. Furthermore, the dermal sinus is incomplete and has no cutaneous manifestations. This represents a novel variant in the spectrum of cranial dermal sinus abnormalities.

\section{Case Report}

A 31-year-old right handed female presented with a new onset seizure. The seizure consisted of sudden staring into space, followed by tonic-clonic activity involving the left leg, left arm, and then generalized secondarily. The patient was amnestic for the event and had post-ictal confusion and urinary incontinence. However, she denied any headaches, nausea, vomiting, weakness, head trauma, or memory problems. Family history was unknown since she was adopted in her first year of life. General and neurological examinations were within normal limits. Complete blood count, serum electrolytes and coagulation studies were normal. The patient was started on dilantin.

The lateral scout view of the computed tomography (CT) of the head (Figure 1) disclosed an elongated irregular skull defect with sclerotic borders involving the temporal region posterior to the coronal suture. The unenhanced axial CT (Figure 2) revealed a lobulated mass predominantly of fat density in the right temporal region. There was no midline shift or edema. Arc-like calcifications were present at the peripheral aspect of the mass. A sinus tract was seen crossing both skull tables and the diploe. The inner table showed hyperostosis bordering the sinus opening. The overlying scalp was entirely normal. Magnetic resonance imaging (MRI) scan (Figure 3) again demonstrated the predominantly fatty nature of the mass and its superior temporal location. Hyperintense signal on T1-weighted images (T1WI) was noted filling the sinus tract. The mass had lower signal on T2-weighted images (T2WI) compared to T1WI. There was no surrounding edema or abnormal contrast enhancement.

The patient underwent a right temporal craniotomy. The shaved scalp was completely normal with no evidence of sinus opening. After reflecting the scalp, a $1.5 \times 0.5 \mathrm{~cm}$ temporal bone defect was noted. The temporal bone defect communicated with the temporal dermoid cyst, via

\footnotetext{
From the Divisions of Neurosurgery (JAS, KMJ) and Neuroradiology (IAA), Montreal General Hospital, McGill University, Montreal, Quebec, Canada RECEIVED APRIL 22, 1999. ACCEPTED IN FINAL FORM AUGUST 3, 1999 Reprint requests to: Karen M. Johnston, Division of Neurosurgery, Montreal General Hospital, 1650 Cedar Avenue, Montreal, Quebec, Canada H3G 1A4
} 


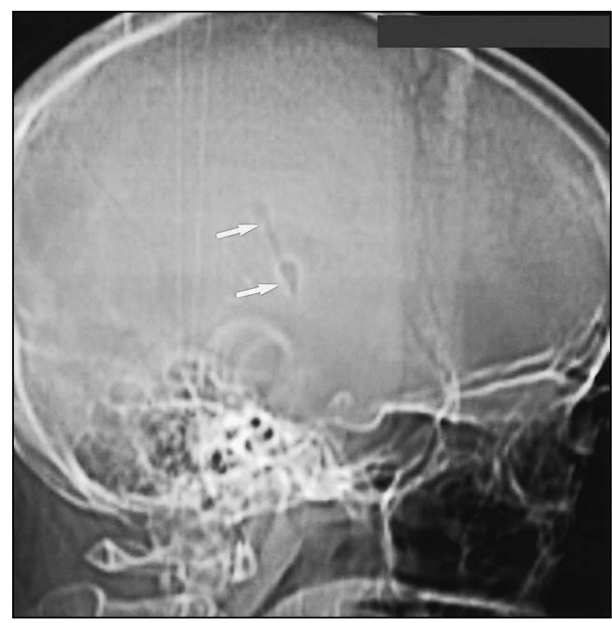

Figure 1: Lateral CT scout view of head showing an elongated irregular defect with sclerotic borders (arrows) involving the temporal region.

a sinus tract, which passed through a dural defect (Figure 4). The cyst was well encapsulated and contained adipose tissue, sebum, and hair. A gross total resection of the cyst and sinus tract was obtained. The inner table of the bony defect was also removed. Routine hematoxylin-eosin (H\&E) staining revealed a typical dermoid cyst with stratified squamous epithelium and poorly differentiated hair follicles. Postoperatively, the patient did well without complications or further seizures. The patient was discharged to home within one week. Post operative CT scan of head at six weeks revealed a complete resection. Follow-up at one year revealed no further seizures.

\section{DISCUSSION}

Dermoid cysts are one of the rarest, benign intracranial mass lesions, with reported frequency between 0.04 to $0.06 \%$ of all intracranial tumors. ${ }^{5,6}$ They most commonly occur along the midline and infratentorially. 5,7 Supratentorial dermoid cysts are a rare subset of cranial dermoid cysts. In one series, only eight patients with supratentorial dermoid cysts were seen over a 37 year period..$^{5}$ Bucciero, ${ }^{8,9}$ presented five patients over a 10 year span. Rubin ${ }^{6}$ reports only four supratentorial dermoid cysts over 11 years. Yasargil ${ }^{10}$ describes only seven supratentorial dermoid cysts over a 21 year period.

Likewise, cranial dermal sinuses are uncommon. In a comprehensive review of the literature on cranial dermal sinuses between 1930 and 1987, French ${ }^{2}$ noted fewer than 100 patients reported in the literature. ${ }^{2,3}$ Similar to dermoid cysts, dermal sinuses most often form along the midline. In French's review, $95 \%$ occurred along the midline $(84 \%$ occipital bone and $11 \%$ at the nasion). ${ }^{2}$ The remaining $5 \%$ were off midline, all of which involved the posterior parietal area. Furthermore, only $16 \%$ of all cranial dermal sinuses occurred supratentorially; (11\% at the nasion, and $5 \%$ in the parietal area). In our case, the cranial dermal sinus was located off midline and supratentorially, in the right superior temporal region.

Supratentorial dermoid cysts rarely have an associated dermal sinus. ${ }^{5,6,8-10}$ This is in direct contrast to infratentorial dermoid cysts which often have an associated dermal sinus. ${ }^{3}$ Supratentorial intracranial dermoid cysts have been reported in association with nasal dermal sinuses. However, only 26 such cases have been reported in the literature before $1993 .{ }^{11}$ A single case has been reported of a supratentorial intradiploic dermoid cyst with an associated dermal sinus. ${ }^{12}$ In our case, the supratentorial dermoid cyst was associated with an off-midline, uniquely incomplete cranial dermal sinus tract. The dermal sinus involved the bone and dura, but not the overlying skin. By definition, a cranial dermal sinus is a tract lined with stratified squamous epithelium that forms a potential communication between the scalp and the deeper tissues within the cranial cavity. $^{2}$ Thus, in our case, this tract is more accurately called a trans-dural, trans-osseous sinus tract since it does not communicate with the skin. To our knowledge, this particular variant has not been previously reported in the English literature. This represents a unique stage in the spectrum of cranial dermal sinus abnormalities.

The exact etiology of dermal sinuses and their associated
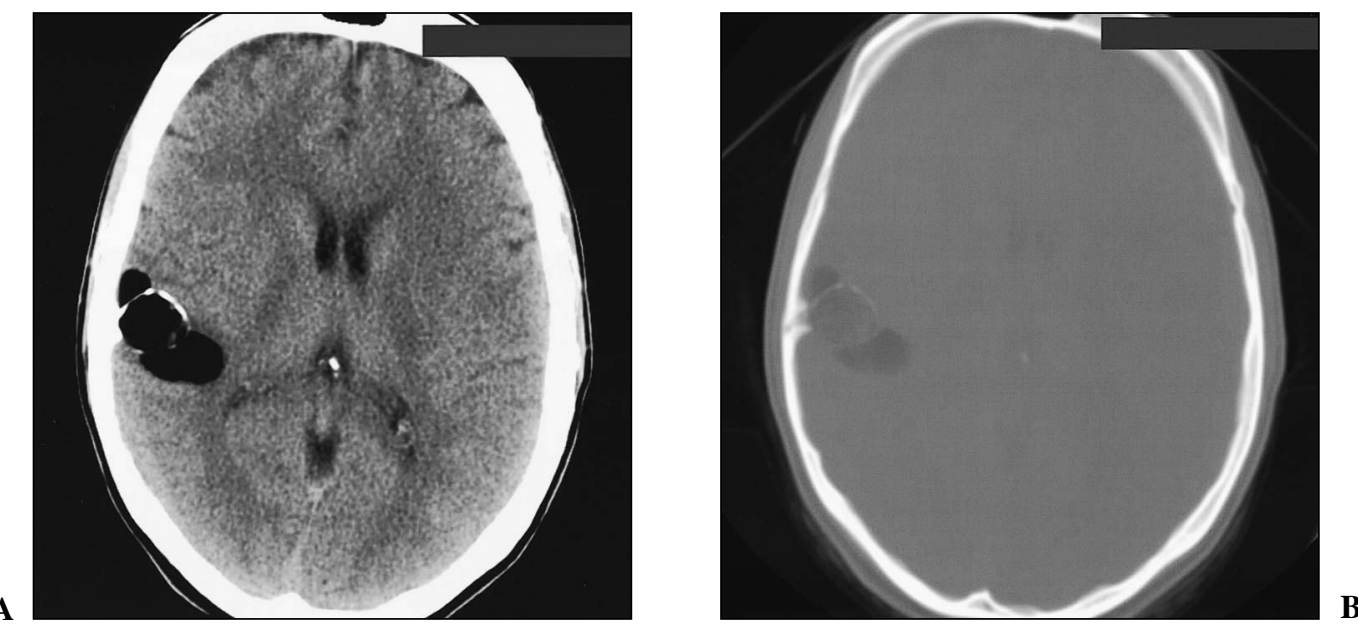

Figure 2: Unenhanced axial CT scan of brain at level of thalami. The soft tissue window (a) demonstrates a lobulated mass predominantly of fat density in the right temporal region. Arc-like calcifications are seen at the periphery of the lesion. The bone window (b) shows a tract traversing both skull tables and diploe with hyperostosis of the inner table. 
A
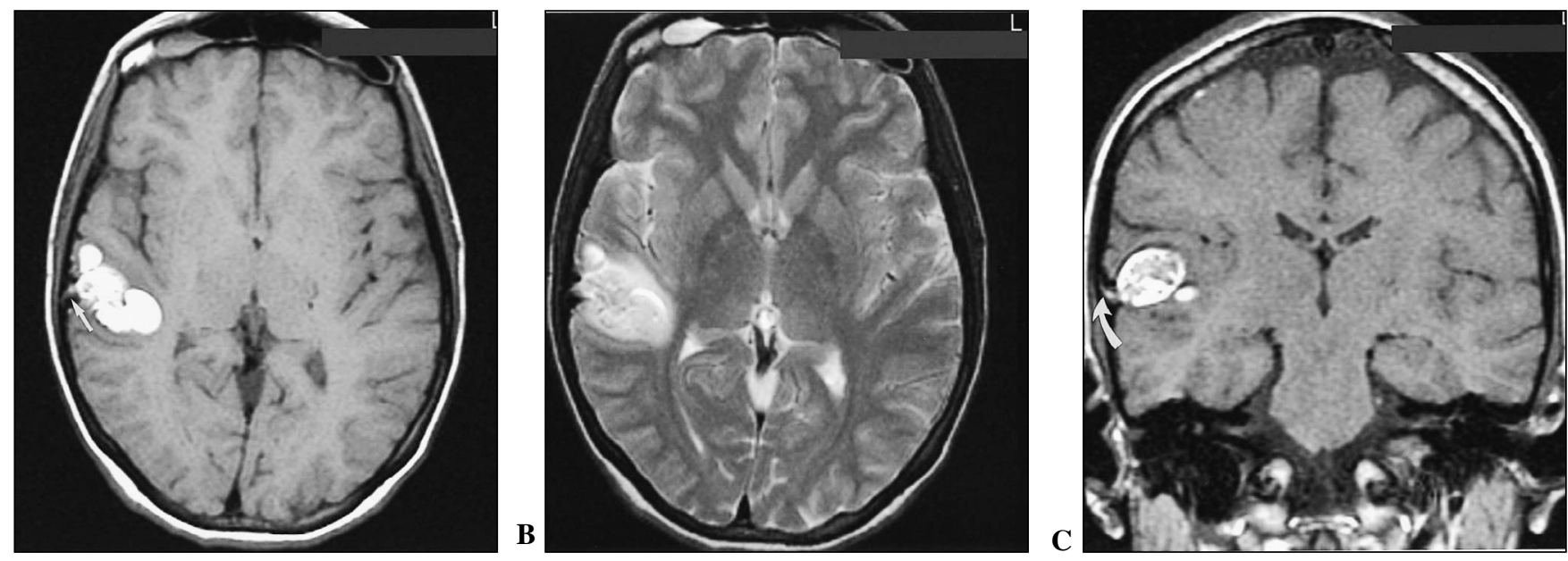

Figure 3: Unenhanced MRI of brain at level of thalami. Axial T1WI (a) shows a lobulated hyperintense lesion in the right temporal lobe. The sinus tract is filled with moderately hyperintense material (arrow). Due to the predominant fatty nature, the signal intensity of the mass is lower on T2WI (b) compared to T1WI. Incidental mucosal thickening is seen in both frontal sinuses with fluid accumulation in the right frontal sinus. Coronal T1WI (c) clearly demonstrates the obliquely oriented sinus tract (arrow) filled with hyperintense material. The mass is well circumscribed and located in the right superior temporal gyrus. Because of different fat content, the heterogeneous central portion of the mass appears less hyperintense than the periphery.

inclusion cysts remains unclear. Since Von Remak ${ }^{13}$ postulated in 1854 that dermoid cysts arise from misplaced epithelial tissues, only modifications of this thesis have been made over the years. These cysts are thought to arise from the ectopic inclusion of epithelial cells during closure of the neural tube between the third and fifth weeks of intrauterine life. Alternatively, others have suggested they arise from enfolding of epithelial cells at the sites of the secondary optic and otic vesicles. ${ }^{2,6}$ Yet others propose that dermoid cysts originate from mesodermal nest cells dysembryogenetically adherent to primitive veins. ${ }^{5}$ In the present case, the dermoid cyst and dermal sinus were located in the right temporal region. Considering the location, it is quite possible that it originated from an enfolding of epithelial cells during lateral migration of the otic vesicles.

The clinical presentation of patients with a dermal sinus depends on several factors. Patients with a dermal sinus communicating with the external environment, tend to present earlier due to central nervous system infection. ${ }^{2}$ However, patients with dermal sinuses, which are incomplete, present significantly later with symptoms attributable to a slowly growing mass lesion. Seizures are a particularly common presenting symptom and are often the only presenting feature, ${ }^{5,7,10}$ as in our case. Supratentorial dermoid cysts can also present by rupturing into an adjacent ventricle causing seizures, or chemical meningitis.,6,7 Associated systemic or neural anomalies are unusual although Klippel-Feil deformity, hemivertebrae, fused vertebrae and hypoplasia of the cerebellar vermis have been described. ${ }^{2}$

Neuroimaging of cranial dermal sinuses and dermoid cysts reveals rather characteristic features. The dermal sinus is often apparent on skull X-ray and bone windows of CT. On CT,

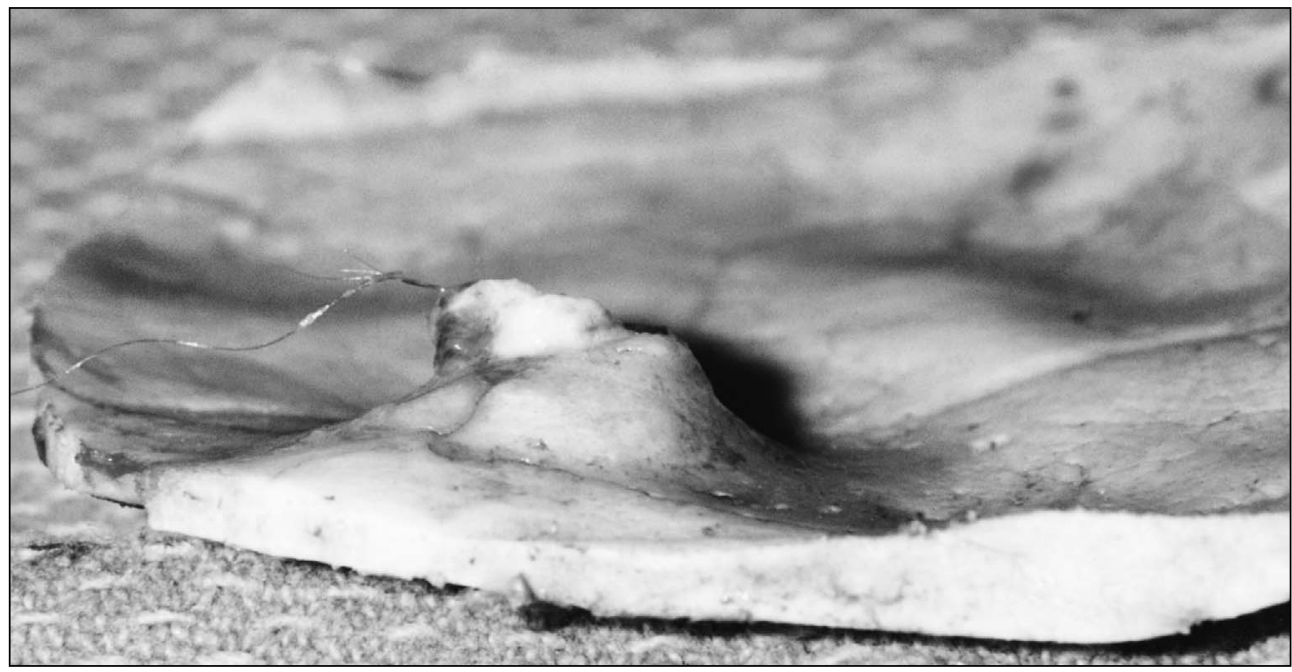

Figure 4: Intraoperative photograph of the temporal bone flap demonstrates hyperostosis of the inner table surrounding the dermal sinus. Adipose and hair contents of the dermal sinus are well illustrated. 
dermoid cysts typically appear as a markedly hypodense round lesion (Houndsfield units from -20 to -40 ) with capsular calcification but no enhancement or cerebral edema. ${ }^{5,6,14}$ On MRI, dermoid cysts have high signal intensity on T1WI and lower signal intensity on T2WI., ${ }^{5,14}$ The contents of the sinus tract are better demonstrated by MRI. In this case, the radiographic differential diagnosis was limited and included a dermal sinus with either a dermoid or terato-dermoid cyst.

\section{Conclusion}

We have presented a patient with a rare superior temporal dermoid cyst and an associated unique dermal sinus tract. Unusual features of the dermal sinus tract include a supratentorial, non-midline location and a uniquely incomplete nature. In this case, the dermal sinus tract involved the skull and dura without involving the overlying skin. This represents a novel variant in the spectrum of cranial dermal sinus abnormalities.

\section{ACKNOWLEDGEMENTS}

The authors are grateful to photographer, John Labelle for taking several intraoperative photographs and to visiting medical student, Michael Ruber for translating Von Remark's paper from German to English.

\section{REFERENCES}

1. Ogle, J. Cases illustrating the formation of morbid growths, deposits, tumors, cysts, etc., in connection with the brain and spinal cord, and their membranes. Br For Med Chir Rev 1865; 36:200-238.

2. French BN. Midline fusion defects and defects of formation. In: Youmans JR, ed. Neurological Surgery. Philadelphia: WB Saunders, 1990: 1164-1169.

3. Logue V, Till K. Posterior fossa dermoid cysts with special reference to intracranial infection. J Neurol Neurosurg Psychiatry 1952; 15: 1-12.

4. Wright RL. Congenital dermal sinuses. Progr Neurol Surg 1971; 4: 175-191.

5. Lunardi P, Missori P. Supratentorial dermoid cysts. J Neurosurg 1991; 75: 262-266.

6. Rubin G, Scienza R, Pasqualin A, Rosta L, Da Pian R. Craniocerebral epidermoids and dermoids. A review of 44 cases. Acta Neurochir (Wien) 1989; 97: 1-16.

7. Tytus JS, Pennybacker J. Pearly tumors in relation to the central nervous system. J Neurol Neurosurg Psychiatry. 1956; 19: 241-259.

8. Bucciero A, Del Basso De Caro ML, Carraturo S. Supratentorial dermoid cysts: presentation and management of five cases. J Neurosurg Sci 1995; 39: 7-11.

9. Bucciero A, De Caro ML, Tedeschi E, et al. Intracranial epidermoid and dermoid cysts: results of microsurgery. Minerva Chir 1997; 52: 863-866

10. Yasargil MG, Abernathey CD, Sarioglu AC. Microneurosurgical treatment of intracranial dermoid and epidermoid tumors. Neurosurgery $1989 ; 24: 561-567$.

11. Brydon HL. Intracranial dermoid cysts with nasal dermal sinuses. Acta Neurochir (Wien) 1992;118: 185-188.

12. Neblett CR, Caram PC, Morris R. Lateral congenital dermal sinus tract associated with an intradiploic dermoid tumor. J Neurosurg 1970; 33: 103-105.

13. Von Remak R. Ein Beitrag zur entwicke-lungs-geschichte der Krebshaften geschwulste. Dt. Klin 1854; 6: 170-174.

14. Gormley WB, Tomecek FJ, Qureshi N, Malik GM. Craniocerebral epidermoid and dermoid tumours: a review of 32 cases. Acta Neurochir (Wein) 1994; 128: 115-121. 